\title{
Dissipative generation of pure steady states and a gambler's ruin problem
}

\author{
Vladislav Popkov, ${ }^{1,2,3}$ Simon Essink, ${ }^{4}$ Corinna Kollath, ${ }^{5}$ and Carlo Presilla ${ }^{6,7}$ \\ ${ }^{1}$ Department of Physics, University of Wuppertal, Gaussstraße20, 42119 Wuppertal, Germany \\ ${ }^{2}$ Institut für Theoretische Physik, Universität Köln, Zülpicher str. 77, Köln, Germany \\ ${ }^{3}$ Faculty of Mathematics and Physics, University of Ljubljana, Jadranska 19, SI-1000 Ljubljana, Slovenia \\ ${ }^{4}$ HISKP, University of Bonn, Nussallee 14-16, 53115 Bonn, Germany \\ ${ }^{5}$ PI, University of Bonn, Nussallee 12, 53115 Bonn, Germany \\ ${ }^{6}$ Dipartimento di Fisica, Sapienza Università di Roma, Piazzale Aldo Moro 2, Roma 00185, Italy \\ ${ }^{7}$ Istituto Nazionale di Fisica Nucleare, Sezione di Roma 1, Roma 00185, Italy
}

\begin{abstract}
We consider an open quantum system, with dissipation applied only to a part of its degrees of freedom, evolving via a quantum Markov dynamics. We demonstrate that, in the Zeno regime of large dissipation, the relaxation of the quantum system towards a pure quantum state is linked to the evolution of a classical Markov process towards a single absorbing state. The rates of the associated classical Markov process are determined by the original quantum dynamics. Extension of this correspondence to absorbing states with internal structure allows us to establish a general criterion for having a Zeno-limit nonequilibrium stationary state of arbitrary finite rank. An application of this criterion is illustrated in the case of an open $X X Z$ spin-1/2 chain dissipatively coupled at its edges to baths with fixed and different polarizations. For this system, we find exact nonequilibrium steady-state solutions of ranks 1 and 2 .
\end{abstract}

PACS numbers: 03.65.Yz, 03.65.Xp, 02.50.-r

\section{INTRODUCTION}

The dynamics of a classical Markov process with an absorbing state, e.g., a so-called gambler's ruin problem [1], stops once the absorbing state is reached, i.e., the gambler has no more coins left. All the other states in which the gambler has a finite number of coins, provided that a bank has an infinite money reserve, are transitory states. Reaching an absorbing state marks the end of the time evolution.

One of our aims is to point out that, surprisingly, the Markovian non-unitary evolution of an open quantum system affected by dissipation towards a pure quantum state can be linked to a classical Markov process with an absorbing state. This link is meaningful and nontrivial if the dissipation acts only on a part of the degrees of freedom of the quantum system and provided that the dissipation is strong, i.e., in the so called quantum Zeno regime [2-4].

Markovian dynamics of an open quantum system is described by a Lindblad master equation (LME), and part of the degrees of freedom corresponding to the eigenstates of the system Hamiltonian evolve via a classical Markov process (MP), the so-called Pauli master equation [5]. This MP, however, does not provide substantial information about the nonequilibrium stationary state (NESS) of the system, since the eigenstates of the system Hamiltonian do not coincide, generically, with those of the NESS. The situation becomes different in the Zeno regime which is governed via an effective Hamiltonian [6, 7], commuting with the Zeno NESS [6]. The quantum Zeno regime is a widely used experimental tool nowadays [8-13], and has applications ranging from engineering dissipative quantum gates [14] and topological states [15] to quantum simulators [16] and universal quantum computations [17-21].

It has been shown in [22], that the Lindblad temporal evolution of the reduced density matrix in the Zeno limit can be described, at the final stage of relaxation, in terms of an auxil- iary classical Markov process, with rates obtainable from the original quantum system. In this auxiliary Markov process, the state probabilities are the populations of the eigenstates of the dissipation-projected Hamiltonian of the quantum system (see later), and converge ultimately to the NESS eigenvalues. Now, suppose that the auxiliary Markov process has an absorbing state, i.e., all populations, except one, vanish in time. Its quantum counterpart, the quantum density matrix, will relax, in time, to a NESS with only one eigenstate being populated, i.e., to a pure quantum state. Conversely, the convergence of a quantum NESS towards a pure state (a rather exceptional scenario, given that a generic quantum state is mixed) implies that the corresponding auxiliary Markov process has an absorbing state.

Exploring further the above analogy is fruitful since we can use the well-developed theory of classical Markov processes with absorbing states [23] for investigating open quantum systems. As a first step, we establish a criterion for a Zeno NESS (assumed unique) to have an arbitrary rank $r$. Our criterion contains a classical part, inherited from the theory of Markov processes with absorbing states, and an intrinsic quantum part. The criterion is formulated in terms of the spectral problem of the dissipation-projected Hamiltonian, which is drastically simpler then the original Liouvillian problem.

We start by outlining our setup and revisiting the connection between the Zeno-limit dynamics of the reduced density matrix and the associated classical MPs. After pointing out a link between a pure NESS (NESS of rank 1) and a MP with an absorbing state, we extend the analogy to a NESS of arbitrary rank, and formulate our criterion. The criterion is then applied to the paradigmatic one-dimensional $X X Z$ spin model with dissipative boundary driving, For this system we find exact Zeno-limit NESS solutions with rank $r=1$ and 2 . 


\section{FINITE RANK NESS AND ITS CONNECTION TO A MARKOV PROCESS}

We consider an open quantum system with Hilbert space $\mathcal{H}$ of finite dimension $d$ and a dissipation acting only on a part of its degrees of freedom, namely, the subspace $\mathcal{H}_{0}$ of dimension $d_{0}$. We assume that the global Hilbert space can be partitioned as a tensor product of $\mathcal{H}_{0}$ and $\mathcal{H}_{1}, \mathcal{H}=\mathcal{H}_{0} \otimes \mathcal{H}_{1}$, where $\mathcal{H}_{1}$ is the remaining part of the Hilbert space not directly coupled to the dissipation. The time evolution of the system is described via the LME [5, 24]

$$
\frac{\partial \rho}{\partial t}=-\frac{i}{\hbar}[H, \rho]+\Gamma \mathcal{D}[\rho] .
$$

Here the Hamiltonian $H$ describes the unitary part of the dynamics, and $\mathcal{D}$ is a Lindbladian dissipator describing the interaction with the environment via Lindblad operators $L_{k}$,

$$
\begin{aligned}
\mathcal{D}[\rho] & =\sum_{k} \mathcal{D}_{L_{k}}[\rho] \\
& =\sum_{k}\left(L_{k} \rho L_{k}^{\dagger}-\frac{1}{2} L_{k}^{\dagger} L_{k} \rho-\frac{1}{2} \rho L_{k}^{\dagger} L_{k}\right) .
\end{aligned}
$$

We assume that the effective dissipation strength $\Gamma$ is much stronger than the unitary part of the evolution, and that the dissipator $\mathcal{D}$ alone targets a unique steady state with the density matrix $\psi_{0}$ in $\mathcal{H}_{0}$, namely, $\mathcal{D} \psi_{0}=0$.

In the limit of large $\Gamma$ and time $t \gg 1 / \Gamma$, the Lindblad dynamics is effectively limited to the decoherence-free subspace, namely, $\rho(t) \approx \psi_{0} \otimes R(t)$. In fact, it has been shown in [22] that, if the dissipator is diagonalizable with spectrum $\mathcal{D} \psi_{n}=\xi_{n} \psi_{n}(n$ labeling different right eigenstates of $\mathcal{D})$, then, for $t \gg 1 / \Gamma,\left\|\rho(t)-\psi_{0} \otimes R(t)\right\|=O(1 / \Gamma)$, where $R(t)$ is determined by the renormalized master equation

$$
\frac{\partial R}{\partial t}=-\frac{i}{\hbar}[\tilde{H}, R]+\frac{1}{\Gamma} \tilde{\mathcal{D}}[R(t)]
$$

where $\tilde{H}$ and $\tilde{\mathcal{D}}$ describe effective unitary and dissipative temporal evolution within the dissipation-free subspace, and can be calculated from $H$ and $\mathcal{D}$ of the original LME (1) with the help of the Dyson expansion with respect to the $1 / \Gamma$ parameter [22]. In general, see [22], $\tilde{H}$, apart from the zeroth-order term $\lim _{\Gamma \rightarrow \infty} \tilde{H}=h_{D}$, can have a $O(1 / \Gamma)$ correction. In the following we set $\hbar=1$. The dissipation projected Hamiltonian $h_{D}$, is given by $[22,25]$

$$
h_{D}=\operatorname{tr}_{\mathcal{H}_{0}}\left[\left(\psi_{0} \otimes I_{\mathcal{H}_{1}}\right) H\right] \text {, }
$$

i.e., $h_{D}$ corresponds to a projection of the original Hamiltonian $H$ on the decoherence-free subspace governed by the kernel $\psi_{0}$ of the dissipator $\mathcal{D}$.

For simplicity, we assume $\tilde{H}=h_{D}$, and expand the steadystate solution of Eq. (3) $R_{\infty}(\Gamma)$ in powers of $1 / \Gamma$,

$$
R_{\infty}=\sum_{m=0}^{\infty} \Gamma^{-m} R_{\infty}^{(m)} .
$$

The series is convergent for sufficiently large $\Gamma$.

We are mainly interested in the final nonequilibrium steady state described by the density matrix $\psi_{0} \otimes R_{\infty}^{(0)}$, where $R_{\infty}^{(0)}=$ $\lim _{\Gamma \rightarrow \infty} \lim _{t \rightarrow \infty} R(t)$.

We insert the ansatz (5) in Eq. (3) for the steady state and compare the orders of $1 / \Gamma^{k}$. The first two orders $k=0,1$ yield

$$
\begin{aligned}
{\left[h_{D}, R_{\infty}^{(0)}\right] } & =0, \\
-i\left[h_{D}, R_{\infty}^{(1)}\right]+\tilde{\mathcal{D}}\left[R_{\infty}^{(0)}\right] & =0 .
\end{aligned}
$$

Note that further orders $1 / \Gamma^{k}$ with $k>1$ cannot be trusted since Eq. (3) itself was obtained up to terms of order $1 / \Gamma$.

Denote by $|\alpha\rangle$ a common eigenbasis of $h_{D}$ and $R_{\infty}^{(0)}$ which is always possible to find since both are Hermitian and commute (6). We write $R_{\infty}^{(0)}$ in this basis as

$$
R_{\infty}^{(0)}=\sum_{\alpha=0}^{d_{1}-1} \nu_{\alpha}^{\infty}|\alpha\rangle\langle\alpha|
$$

where $d_{1}$ is the dimension of the subspace $\mathcal{H}_{1}$. Let us now rewrite Eq. (7) as a set of scalar equations using the basis $|\alpha\rangle$, namely

$$
\begin{gathered}
Q_{\alpha \alpha^{\prime}} \equiv\left\langle\alpha\left|\tilde{\mathcal{D}}\left[R_{\infty}^{(0)}\right]-i\left[h_{D}, R_{\infty}^{(1)}\right]\right| \alpha^{\prime}\right\rangle=0 \\
\alpha, \alpha^{\prime}=0, \ldots, d_{1}-1
\end{gathered}
$$

We postpone an analysis of the complete set $Q_{\alpha \alpha^{\prime}}=0$ and first look at diagonal subset $Q_{\alpha \alpha}=0$, for all $\alpha$. Assuming the effective dissipator $\tilde{\mathcal{D}}$ to be of the canonical form $\tilde{\mathcal{D}}$. $=$ $\sum_{k} \gamma_{k}\left(\tilde{L}_{k} \cdot \tilde{L}_{k}^{\dagger}-\frac{1}{2}\left\{\cdot, \tilde{L}_{k}^{\dagger} \tilde{L}_{k}\right\}\right)$, we obtain, after some algebra, a closed set of equations for $\nu_{\alpha}^{\infty}$,

$$
\begin{aligned}
& \sum_{\beta \neq \alpha}^{d_{1}-1} w_{\beta \alpha} \nu_{\beta}^{\infty}-\nu_{\alpha}^{\infty} \sum_{\beta \neq \alpha}^{d_{1}-1} w_{\alpha \beta}=0, \\
& w_{\beta \alpha}=\frac{1}{\Gamma} \sum_{k} \gamma_{k}\left|\left\langle\alpha\left|\tilde{L}_{k}\right| \beta\right\rangle\right|^{2} .
\end{aligned}
$$

We recognize in (10) a steady master equation of a Markov process with transition rates $w_{\alpha \beta}$ between the states $\alpha$ and $\beta$. The factor $\frac{1}{\Gamma}$ in Eq. (11) signals that the relaxation towards the steady-state slows down with an increased dissipation strength, see $[6,22]$. The Perron-Frobenius theorem guarantees the existence of a solution of Eq. (10) with nonnegative entries $\nu_{\alpha}^{\infty}$, which, according to (8), have the physical meaning of the eigenvalues of the reduced density matrix in the Zeno limit $R_{\infty}^{(0)}$, obeying the normalization condition $\sum_{\alpha} \nu_{\alpha}^{\infty}=1$

In the following we establish a criterion for a Zeno NESS $R_{\infty}^{(0)}$ to have a reduced rank $r<d_{1}$. We start with the case of pure NESS corresponding to $r=1$, when $R_{\infty}^{(0)}=|0\rangle\langle 0|$ and generalize it afterwards. We remark that this case was already considered in [26], but now we revisit it using the stochastic interpretation. 


\section{PURE NESS}

A NESS of the form $R_{\infty}^{(0)}=|0\rangle\langle 0|$, implies the existence of a unique steady state solution of the Markov equation (10). This means that for all $\beta=0, \ldots, d_{1}-1$, the solution fulfills $\nu_{\beta}^{\infty}=\delta_{0, \beta}$. In the stochastic Markov picture, a unique Markov process steady state of the form $\nu_{\beta}^{\infty}=\delta_{0, \beta}$ can arise if and only if the state $\alpha=0$ is an absorbing state of the Markov process. This means that the system cannot escape from state zero, namely,

$$
w_{0 \alpha}=0, \quad \text { for any } \alpha>0,
$$

while all the other states of the Markov process $\alpha>0$ are transient. Thus, we establish a link between a pure NESS and an auxiliary classical Markov process with an absorbing state.

\section{NESS OF AN ARBITRARY REDUCED RANK $r$}

Analogously, we interpret a Zeno NESS of rank $r$ smaller than the full rank, $r<d_{1}$,

$$
R_{\infty}^{(0)}=\sum_{z=0}^{r-1} \nu_{z}^{\infty}|z\rangle\langle z|
$$

as a consequence of the existence of a closed subset of states in the auxiliary classical Markov process with rates $w_{\alpha \beta}$ satisfying closed set condition $w_{z \alpha}=0$, for all $z<r$ and $\alpha \geq r$. At this point, however, one should not forget that our original problem is an intrinsic quantum problem, not describable by just set of eigenvalues $\nu_{z}^{\infty}$. In particular, we have up to now neglected the conditions arising from off-diagonal values of $Q_{\alpha, \alpha^{\prime}}$. What is the full set of conditions which guarantee the Zeno NESS to have a reduced rank? We postulate that an answer to this question is given by the following criterion:

\section{A. Criterion}

- Let $|\alpha\rangle$ be an eigenbasis of the dissipation-projected Hamiltonian $h_{D}, \lambda_{\alpha}$ being the respective eigenvalues, and let $\tilde{\mathcal{D}} \cdot=\sum_{k} \gamma_{k}\left(\tilde{L}_{k} \cdot \tilde{L}_{k}^{\dagger}-\frac{1}{2}\left\{\cdot, \tilde{L}_{k}^{\dagger} \tilde{L}_{k}\right\}\right)$ be the Lindbladian of the effective dynamics. A state $\rho_{\infty}=\psi_{0} \otimes \sum_{z=0}^{r-1} \nu_{z}^{\infty}|z\rangle\langle z|$ is a NESS state of Eq. (1) in the Zeno limit, if and only if the following three conditions are satisfied:

(A) states $z=0,1, \ldots, r-1$ form a closed ergodic set in the associated Markov process, defined via the transition rates $w_{\alpha \beta}=\sum_{k} \gamma_{k}\left|\left\langle\beta\left|\tilde{L}_{k}\right| \alpha\right\rangle\right|^{2} / \Gamma$

(B) all the other states $r, r+1, \ldots, d_{1}-1$ are transient;

(C) for any state $|\alpha\rangle$ such that $\lambda_{\alpha}=\lambda_{z}$, where $\alpha$ belongs to the transient set and $z$ belongs to the closed set, we have $\left\langle z\left|\sum_{k} \gamma_{k} \tilde{L}_{k}^{\dagger} \tilde{L}_{k}\right| \alpha\right\rangle=0$.

Before proceeding to justify the criterion, we note that conditions $\mathrm{A}$ and $\mathrm{B}$ are rooted in the associated classical Markov process, while condition $\mathrm{C}$ has an intrinsically quantum origin. Let us note that for the Zeno-limit NESS to be a pure state $(r=1)$ necessary and sufficient conditions were verified by an alternative method in [26]. The conditions given in [26] are equivalent to our conditions $\mathrm{A}, \mathrm{B}$ and $\mathrm{C}$ for $r=1$ which prove the criterion for $r=1$.

\section{B. Proof of the necessity of the criterion.}

. In brief, conditions A and B, together with the stochasticity property of the transition matrix, provide the existence of a unique steady-state solution of the associated Markov process, with $\nu_{0}, \nu_{1}, \ldots, \nu_{r-1}$ nonzero and $\nu_{k}=0$, for $k \geq r$.

To see this, consider the full set of scalar equations in (9). Noticing that $\left\langle\alpha\left|\left[h_{D}, R_{\infty}^{(1)}\right]\right| \alpha\right\rangle=0$ and using Eq. (13), we find that the equations $Q_{\alpha \alpha}=0$ for $\alpha \geq r$ are trivially satisfied. The equations $Q_{z z}=0$, with $z=0,1, \ldots, r-1$ yield a steady-state master equation (10) for steady-state probabilities $\nu_{z}^{\infty}$ inside the closed set.

Condition $\mathrm{C}$ enters the criterion only if the Hamiltonian $h_{D}$ has a special degeneracy, namely, $\lambda_{\alpha}=\lambda_{z}$, where $z$ belongs to the closed set and $\alpha$ belongs to the transient one.

The off-diagonal conditions in Eq. (9), namely, $Q_{z \alpha}=0$, with $z \leq r-1$ and $\alpha \geq r$, yield, after some algebra,

$$
-i\left(\lambda_{z}-\lambda_{\alpha}\right)\left\langle z\left|R_{\infty}^{(1)}\right| \alpha\right\rangle=\frac{\nu_{z}^{\infty}}{2}\left\langle z\left|\sum_{k} \gamma_{k} \tilde{L}_{k}^{\dagger} \tilde{L}_{k}\right| \alpha\right\rangle
$$

In deriving Eq. (14) we used the properties for $\alpha \geq r$ $R_{\infty}^{(0)}|\alpha\rangle=0$ and $\left\langle z\left|\tilde{L}_{n}^{\dagger}\right| \alpha\right\rangle=0$. The latter follows from the closed set property A, namely, $w_{z \alpha}=\sum_{n} \gamma_{n}\left|\left\langle\alpha\left|\tilde{L}_{n}\right| z\right\rangle\right|^{2}=$ 0 . Note that by virtue of our assumption (13) we have $\nu_{z}^{\infty} \neq$ 0 , thus, if $\lambda_{z}=\lambda_{\alpha}$, the right-hand side of Eq. (14) must vanish, yielding condition $\mathrm{C}$ of the criterion. In conclusion, conditions $\mathrm{A}, \mathrm{B}$ and $\mathrm{C}$ are indeed necessary for the NESS in the Zeno limit to have rank $r$.

\section{Argument for sufficiency of the criterion.}

Whereas the necessity of the conditions in the criterion is proven, the question of sufficiency appears more delicate. For the pure state case, $r=1$, sufficiency of our criterion has been rigorously proved in [26]. For higher ranks $r>1$ we do not have a rigorous argument so far. Instead, here we checked our criterion for $r=2$ numerically for a specific model, dissipatively driven spin chains.

\section{Reformulation of conditions A and B.}

In the following we show how to check conditions A and B given the rates $w_{\alpha \beta}$.

Condition $\mathrm{A}$ is equivalent to

$$
w_{z \alpha}=0, \quad \text { for all } z \leq r-1 \text { and } \alpha \geq r,
$$

complemented with the requirement of ergodicity: each state within the closed set is reachable from any other state in the closed set in a finite number of steps. 
In order to check condition $\mathrm{B}$, write the transition matrix $F$ (the matrix with elements $F_{\beta \alpha}=w_{\alpha \beta}$ for $\beta \neq \alpha, F_{\alpha \alpha}=$ $-\sum_{\beta \neq \alpha} F_{\beta \alpha}$ ) satisfying the closed set conditions (15) in the canonical form [23]

$$
\begin{aligned}
F & =\left(\begin{array}{cccccc}
\mathcal{X}_{00} & \ldots & \mathcal{X}_{0, r-1} & \mathcal{R}_{0, r} & \ldots & \mathcal{R}_{0, d_{1}-1} \\
\vdots & \vdots & \vdots & \vdots & \vdots & \vdots \\
\mathcal{X}_{r-1,0} & \ldots & \mathcal{X}_{r-1, r-1} & \mathcal{R}_{r-1, r} & \ldots & \mathcal{R}_{r-1, d_{1}-1} \\
0 & \ldots & 0 & \mathcal{K}_{r r} & \ldots & \mathcal{K}_{r, d_{1}-1} \\
\vdots & \vdots & \vdots & \vdots & \vdots & \vdots \\
0 & \ldots & 0 & \mathcal{K}_{d_{1}-1, r} & \ldots & \mathcal{K}_{d_{1}-1, d_{1}-1}
\end{array}\right) \\
& =\left(\begin{array}{cc}
\mathcal{X} & \mathcal{R} \\
0 & \mathcal{K}
\end{array}\right),
\end{aligned}
$$

where $\mathcal{X}_{i i}=-\sum_{j \neq i} \mathcal{X}_{j i}$ and $\mathcal{K}_{i i}=-\sum_{j \neq i} \mathcal{K}_{j i}-$ $\sum_{j} \mathcal{R}_{j i}$. The steady-state equation $\sum_{\beta=0}^{d-1} F_{\alpha \beta} \nu_{\beta}^{\infty}=$ 0 has an obvious closed set solution, namely, $\nu^{\infty}=$ $\left(\nu_{0}^{\infty}, \nu_{1}^{\infty}, \ldots, \nu_{r-1}^{\infty}, 0,0, \ldots, 0\right)$, where the nonzero components $\nu_{z}^{\infty}$ satisfy $\sum_{z^{\prime}=0}^{r-1} \mathcal{X}_{z z^{\prime}} \nu_{z^{\prime}}^{\infty}=0$. This solution is unique if and only if all the other states are transient, which is equivalent to the absence of zero eigenvalues in the spectrum of $\mathcal{K}$, i.e.,

$$
\operatorname{det} \mathcal{K} \neq 0
$$

Indeed, assume $\operatorname{det} \mathcal{K} \neq 0$ and split the equations $\sum_{\beta=0}^{d_{1}-1} F_{\alpha \beta} \nu_{\beta}^{\infty}=0$ in two sets,

$$
\sum_{z^{\prime}=0}^{r-1} \mathcal{X}_{z z^{\prime}} \nu_{z^{\prime}}^{\infty}+\sum_{\alpha=r}^{d_{1}-1} \mathcal{R}_{z \alpha} \nu_{\alpha}^{\infty}=0, \quad z=0,1, \ldots, r-1
$$$$
\sum_{\beta=r}^{d_{1}-1} \mathcal{K}_{\alpha \beta} \nu_{\beta}^{\infty}=0, \quad \alpha=r, r+1, \ldots, d_{1}-1 .
$$

By the assumption (17), Eq. (19) has only the trivial solution $\nu_{\beta}^{\infty}=0$, for all $\beta>0$. Thus the closed set solution is the only solution of Eqs. (18) and (19). Conversely, if $\operatorname{det} \mathcal{K}=0$, then a nontrivial solution of Eq. (19) exists, and, due to the PerronFrobenius theorem, it has non negative real entries. This corresponds to the existence of another closed set of states within the "transient" set of states $\alpha=r, r+1, \ldots, d_{1}-1$, and, therefore, to a violation of the transient hypothesis. Consistency of Eq. (18) is guaranteed by the decomposition theorem for finite Markov chains [23].

\section{EXAMPLE OF A DISSIPATIVELY DRIVEN ONE-DIMENSIONAL $X X Z$ SPIN CHAIN}

In the following, our findings are illustrated with examples based on dissipatively driven one-dimensional $X X Z$ spin chains.

Consider a chain with $N$ sites occupied by spins $s=1 / 2$ and described by the anisotropic Hamiltonian

$$
H=\sum_{n=1}^{N-1} \vec{\sigma}_{n} \cdot\left(J \vec{\sigma}_{n+1}\right)
$$

where $\vec{\sigma}_{n}=\left(\sigma_{n}^{x}, \sigma_{n}^{y}, \sigma_{n}^{z}\right)$ is the vector of the Pauli matrices at site $n$ and $J=\operatorname{diag}\left(J_{x}, J_{y}, J_{z}\right)$ the anisotropy tensor of the exchange interaction. We choose a dissipation acting at the boundary sites 1 and $N$, and targeting two arbitrary single qubit states

$$
\begin{aligned}
& \rho_{L, R}=\mu_{L, R} \vec{n}\left(\theta_{L, R}, \varphi_{L, R}\right) \vec{\sigma}_{1, N} \\
& \vec{n}(\theta, \varphi)=(\sin \theta \cos \varphi, \sin \theta \sin \varphi, \cos \theta),
\end{aligned}
$$

where $\mu_{L, R} \vec{n}_{L, R}$ with $\vec{n}_{L, R} \equiv \vec{n}\left(\theta_{L, R}, \varphi_{L, R}\right)$ are the target polarizations. Here, $L(R)$ refers to the leftmost (righmost) site of the chain. Specifically, introducing $\vec{n}_{L, R}^{\prime}=$ $\vec{n}\left(\frac{\pi}{2}-\theta_{L, R}, \varphi_{L, R}+\pi\right)$ and $\vec{n}_{L, R}^{\prime \prime}=\vec{n}\left(\frac{\pi}{2}, \varphi_{L, R}+\frac{\pi}{2}\right)$, the above dissipation is realized by applying two Lindblad operators at both sites $1, N$ of the chain

$$
\begin{aligned}
& L_{1,2}^{\mathrm{L}}=(2 \sqrt{2})^{-1} \sqrt{1 \pm \mu_{L}}\left(\vec{n}_{L}^{\prime} \mp i \vec{n}_{L}^{\prime \prime}\right) \vec{\sigma}_{1} \\
& L_{1,2}^{\mathrm{R}}=(2 \sqrt{2})^{-1} \sqrt{1 \pm \mu_{R}}\left(\vec{n}_{R}^{\prime} \mp i \vec{n}_{R}^{\prime \prime}\right) \vec{\sigma}_{N} .
\end{aligned}
$$

A realization of the Lindblad dynamics (1) for the present class of models can for example be obtained via a protocol of repeated interactions: the edge spins of the lattice are brought into interaction with two "bath" qubits, the latter qubits being kept in fixed, mixed, or pure states $\rho_{L}$ and $\rho_{R}$ from (21); the interaction has strength $\gamma_{B}$ and repeats periodically at time intervals $\tau$ each time with a bath qubit in the fixed state. Within this protocol, the time evolution from time $t$ to time $t+\tau$ is described by the map

$$
\rho_{t+\tau}=\operatorname{tr}_{0, N+1}\left(e^{-i \mathcal{H} \tau} \rho_{L} \otimes \rho_{t} \otimes \rho_{R} e^{i \mathcal{H} \tau}\right),
$$

where sites $0, N+1$ denote the qubits from the left and right baths, respectively, and $\mathcal{H}=H+\gamma_{B}\left(U_{0,1}+U_{N, N+1}\right)$ is the Hamiltonian of the original system plus the system-bath interactions $U_{0,1}$ and $U_{N, N+1}$. The LME dynamics (1) follows from the above discrete map in the limit $\tau \rightarrow 0, \gamma_{B}^{2} \tau \rightarrow \Gamma$ (see [27]).

In [22] we have demonstrated that in the Zeno limit the effective dynamics of the spin model is described by a LME of type (3) for $R(t)=\operatorname{tr}_{1, N} \rho(t)$, with $\tilde{H}=h_{D}$ and $\tilde{\mathcal{D}}=\tilde{\mathcal{D}}_{L}+\tilde{\mathcal{D}}_{R}$ given by

$$
\begin{aligned}
& h_{D}=\sum_{j=1}^{M-1} \vec{\sigma}_{j} \cdot\left(J \vec{\sigma}_{j+1}\right)+\mu_{L}\left(J \vec{n}_{L}\right) \cdot \vec{\sigma}_{1}+\mu_{R}\left(J \vec{n}_{R}\right) \cdot \vec{\sigma}_{M}, \\
& \tilde{\mathcal{D}}_{L}=2\left(1+\mu_{L}\right) D_{g_{1 L}}+2\left(1-\mu_{L}\right) D_{g_{1 L}^{\dagger}}+\frac{1}{2}\left(1-\mu_{L}^{2}\right) D_{g_{3 L}}, \\
& \tilde{\mathcal{D}}_{R}=2\left(1+\mu_{R}\right) D_{g_{1 R}}+2\left(1-\mu_{R}\right) D_{g_{1 R}^{\dagger}}+\frac{1}{2}\left(1-\mu_{R}^{2}\right) D_{g_{3 R}} .
\end{aligned}
$$

Here the operators act in $\mathcal{H}_{1}$, the Hilbert space of the inner spins $2,3, \ldots, N-1$. We renumerate the inner spins as $1, \ldots, M=N-2$. The operators $D_{g}$ are Lindblad dissipators of standard form defined in terms of the effective Lindblad operators $g_{1 L}=\left(J \vec{n}_{L}^{\prime}\right) \cdot \vec{\sigma}_{1}-i\left(J \vec{n}_{L}^{\prime \prime}\right) \cdot \vec{\sigma}_{1}, g_{3 L}=2\left(J \vec{n}_{L}\right) \cdot \vec{\sigma}_{1}$, $g_{1 R}=\left(J \vec{n}_{R}^{\prime}\right) \cdot \vec{\sigma}_{M}-i\left(J \vec{n}_{R}^{\prime \prime}\right) \cdot \vec{\sigma}_{M}$ and $g_{3 R}=2\left(J \vec{n}_{R}\right) \cdot \vec{\sigma}_{M}$. 


\section{PURE STATE $r=1$}

As a first example of application of our finite rank Zeno NESS criterion, we consider a NESS being a pure spin-helix state (SHS) $\rho_{\infty}=|\xi\rangle\langle\xi|$, where

$$
|\xi\rangle=\bigotimes_{k=1}^{N}\left(\begin{array}{c}
\cos \left(\frac{\theta}{2}\right) e^{-\frac{i}{2} \varphi(k-1)} \\
\sin \left(\frac{\theta}{2}\right) e^{\frac{i}{2} \varphi(k-1)}
\end{array}\right) .
$$

This state describes a frozen wave-like spin structure, formed by a rotation of a local spin around the $z$-axis along the chain, with constant azimuthal angle difference $\varphi$ between neighboring spins. Indeed, the expectation value of the local spin at site $k$ is

$$
\left\langle\xi\left|\vec{\sigma}_{k}\right| \xi\right\rangle=(\sin \theta \cos [\varphi(k-1)], \sin \theta \sin [\varphi(k-1)], \cos \theta) .
$$

We rewrite the factorized NESS (23) in the form evidencing left and right dissipation target states, namely, $\rho_{\infty}=\psi_{0}^{L} \otimes$ $|0\rangle\langle 0| \otimes \psi_{0}^{R}$. This corresponds to choosing $\mu_{L}=\mu_{R}=1$, $\theta_{L}=\theta_{R}=\theta, \varphi_{L}=0, \varphi_{R}=\varphi(N-1)$ in Eq. (21), and

$$
|0\rangle=\bigotimes_{k=1}^{M}\left(\begin{array}{c}
\cos \left(\frac{\theta}{2}\right) e^{-\frac{i}{2} \varphi k} \\
\sin \left(\frac{\theta}{2}\right) e^{\frac{i}{2} \varphi k}
\end{array}\right) .
$$

For the given values of $\mu_{L}$ and $\mu_{R}$ the original Lindbladian dissipator has two nonzero Lindblad operators $L_{1}^{L}$ and $L_{1}^{R}$, and the target boundary states are pure (target mixed boundary states would typically lead to a full rank Zeno NESS). The corresponding effective Lindblad operators $\tilde{L}_{1}=g_{1 L}$ and $\tilde{L}_{2}=g_{1 R}$. The associated effective dissipator is

$$
\tilde{\mathcal{D}}=4 \mathcal{D}_{g_{1 L}}+4 \mathcal{D}_{g_{1 R}} .
$$

In $[28,29]$ it has been shown that Eq. (23) is the exact Zeno-limit NESS of the boundary driven $X X Z$ spin chain with anisotropy $J_{x}=J_{y}=J_{z} / \Delta$, provided $\Delta=\cos \varphi$, targeted single spin states $\psi_{0}^{L, R}$ fit the SHS (23), and (23) does not contain collinear spins.

We now turn to discuss the connection to the Markov process. The stochastic transition matrix of the auxiliary Markov process is defined via the rates $w_{\alpha \beta}$ given by Eq. (11), where $|\alpha\rangle$ and $|\beta\rangle$ are the eigenvectors of $h_{D}$. We thus find

$$
w_{\alpha \beta}=\frac{4}{\Gamma}\left(\left|\left\langle\beta\left|g_{1 L}\right| \alpha\right\rangle\right|^{2}+\left|\left\langle\beta\left|g_{1 R}\right| \alpha\right\rangle\right|^{2}\right) .
$$

One can check that (24) is an eigenstate of $h_{D}$ [with eigenvalue $\lambda_{0}=(N-1) J \cos \varphi$ ], and of $g_{1 R}, g_{1 L}$ with eigenvalues $\pm \kappa, \kappa=i J \sin \theta \sin \varphi$. Using the orthogonality of the eigenbasis of $h_{D}$, we get $\left\langle\alpha\left|g_{1 L}\right| 0\right\rangle=\left\langle\alpha\left|g_{1 R}\right| 0\right\rangle=0$ for all $\alpha>0$, leading [see Eq. (26)] to the absorbing state condition (12). The relaxation of the global reduced density matrix towards a pure NESS thus corresponds, in the language of the auxiliary Markov process, to a convergence of the classical Markov process towards an absorbing state. Furthermore, it has been proven in [26] via a different method that in the Zeno limit the NESS converges to the pure state $|\xi\rangle\langle\xi|$ if and only if Eq. (17) and condition $\mathrm{C}$ are satisfied, in accordance with the criterion for $r=1$.

\section{NESS OF RANK $r=2$}

Here we apply our criterion to find a Zeno-limit NESS of rank 2 , in the same model. We can set the parameters in such a way that condition A for $r=2$ is satisfied, namely, there exists a closed set of two states in the associated Markov process. Choose the first and the last spin of the chain to be dissipatively projected into parallel or antiparallel states, $\varphi_{R}-\varphi_{L}=n \pi$, while keeping $\theta_{L}=\theta_{R}=\theta$, and set $\Delta=\cos \varphi=\cos (2 m \pi /(N-1))$ for parallel orientation and $\Delta=\cos \varphi=\cos ((\pi+2 m \pi) /(N-1))$ for antiparallel orientation. Then, two eigenstates of the dissipation-projected Hamiltonian can be found, namely, the state $\left|0_{+}\right\rangle$given by Eq. (24) and the state $\left|0_{-}\right\rangle$obtained from $\left|0_{+}\right\rangle$by changing the sign of the helicity, $\varphi \rightarrow-\varphi$. Both eigenstates $\left|0_{ \pm}\right\rangle$have the same eigenvalue $\lambda_{0}=(N-1) J \cos \varphi$.

The states $\left|0_{ \pm}\right\rangle$are in general not orthogonal, $\left\langle 0_{-} \mid 0_{+}\right\rangle=$ $\eta$, where $\eta$ can be real or imaginary, $\eta^{*}= \pm \eta$, depending on the parameters. The overlap $\eta$ can be expressed via a $q$ Pochhammer symbol,

$$
\begin{aligned}
& \eta(\theta, \varphi, N)=\prod_{k=1}^{N-2}(\cos (k \varphi)-i \cos (\theta) \sin (k \varphi)) \\
& =e^{-i \frac{M(M+1)}{2} \varphi} \cos ^{2 M}\left(\frac{\theta}{2}\right)\left(-\tan ^{2} \frac{\theta}{2} ; e^{2 i \varphi}\right)_{M} .
\end{aligned}
$$

Out of the states $\left|0_{ \pm}\right\rangle$, we build the orthonormal combinations $|0\rangle=1 / \eta_{+}\left[\left|0_{+}\right\rangle+(\eta /|\eta|)\left|0_{-}\right\rangle\right],|1\rangle=1 / \eta_{-}\left[\left|0_{+}\right\rangle-\right.$ $\left.(\eta /|\eta|)\left|0_{-}\right\rangle\right]$, where $\eta_{ \pm}=\sqrt{2 \pm 2|\eta|}$. Using the fact that $\left|0_{+}\right\rangle$is an eigenstate of $g_{1 R}$ and $g_{1 L}$ with eigenvalue $\kappa$ and $-\kappa$, respectively, we find

$$
\begin{array}{llrl}
g_{1 R}|0\rangle & =-g_{1 L}|0\rangle=a_{0}|1\rangle, & & a_{0}=\kappa \eta_{-} / \eta_{+}, \\
g_{1 R}|1\rangle=-g_{1 L}|1\rangle=a_{1}|0\rangle, & & a_{1}=\kappa \eta_{+} / \eta_{-} .
\end{array}
$$

It follows that the rates of the associated effective Markov process are

$$
\begin{aligned}
& w_{0 \alpha}=w_{1 \alpha}=0, \quad \alpha \geq 2, \\
& w_{01}=\frac{8}{\Gamma}\left|a_{0}\right|^{2}, \\
& w_{10}=\frac{8}{\Gamma}\left|a_{1}\right|^{2} .
\end{aligned}
$$

Equation (30) corresponds to the closed set conditions (15) for the states with labels $z=0,1$, while $w_{10} w_{0,1} \neq 0$ provides the ergodicity of the closed set. Moreover, the system $F \nu^{\infty}=0$, equivalent to $\nu_{0}^{\infty} w_{01}=\nu_{1}^{\infty} w_{10}$, is solved by $\nu_{0}^{\infty} / \nu_{1}^{\infty}=w_{10} / w_{01}=(1+|\eta|)^{2} /(1-|\eta|)^{2}$ and $\nu_{2}^{\infty}=$ $\nu_{3}^{\infty}=\cdots=0$

The explicit form of the rank 2 state, that appears in the Zeno limit is

$$
\rho_{\infty}=\psi_{0}^{L} \otimes\left(\frac{(1+|\eta|)^{2}}{2+2|\eta|^{2}}|0\rangle\left\langle 0\left|+\frac{(1-|\eta|)^{2}}{2+2|\eta|^{2}}\right| 1\right\rangle\langle 1|\right) \otimes \psi_{0}^{R},
$$

with $\psi_{0}^{L, R}=\rho_{L, R}$. The topological aspects of the state (33) are discussed in [30]. Let us note that for $\theta=\pi / 2$ and all 
cases in which this state is the NESS (see below) the overlap $\eta$ simplifies to

$$
\eta= \begin{cases}2^{2-N}, & \text { if } \varphi_{R}-\varphi_{L}=0 \text { and } N \text { even } \\ -(i / 2)^{N-2}, & \text { if } \varphi_{R}-\varphi_{L}= \pm \pi \text { and } N \text { even. }\end{cases}
$$

Finally, we need to check conditions B and C. Unlike condition $\mathrm{A}$, we check them numerically, diagonalizing $h_{D}$. The results for NESS ranks obtained for chains with size $3 \leq N \leq$ 13 are summarized in Table I. Firstly, we notice that the NESS has rank $r=2$ if and only if conditions A-C are satisfied, numerically supporting the validity of our criterion.

In addition, analyzing Table I we notice that a NESS with rank $r=2$ occurs if and only if

$$
\begin{aligned}
& \varphi=(\pi m) /(N-1) \\
& \text { and } N-1 \text { is not a multiple of } m .
\end{aligned}
$$

This pattern has simple geometrical interpretation, namely, the states (23), which define (33), do not contain any pairs of collinear spins, except for the two boundary spins, which are parallel by construction. E.g. for $N=9$ a rank 2 state only appears in the Zeno limit when $\Delta=\cos [(2 \pi m) / 8]$ with $m=1,3,5,7$. If $N-1$ is a prime number, (36) is satisfied for all $\Delta=\cos [(2 \pi m) /(N-1)] m=1,2, \ldots, N-2$.

\section{CONCLUSIONS}

We have established a link between a quantum dissipative NESS with reduced rank and an auxiliary classical Markov process with absorbing states (closed sets). This link paves the way for studies of quantum master equations using the well developed theory of classical Markov processes. In the present paper, using this link, we suggested a criterion for a NESS in the Zeno limit to have a reduced rank. The criterion is illustrated with an example in which rank 1 (pure NESS) and rank 2 NESS solutions appear in dissipatively boundary driven $X X Z$ spin chains . Noteworthy, our criterion has a "classical Markov process" part, (properties A and B), and an intrinsic quantum part (property C), which has no classical analog, being related to a degeneracy of a special eigenvalue in the spectrum of the associated dissipation-projected Hamiltonian. Deep understanding of the quantum part of the criterion remains a challenge for the future. From the applicative viewpoint, our criterion allows dissipative targeting of pure states or simple mixtures of few quantum states, a task of fundamental importance in the initialization of quantum simulators [32] (see also [33] for an up-to-date review of recent and ongoing experiments).

\section{ACKNOWLEDGMENTS}

VP thanks the Department of Physics of Sapienza University of Rome for hospitality and financial support. Financial support from the Deutsche Forschungsgemeinschaft through DFG projects KL 645/20-1 and KO 4771/3-1, projects 277625399 - TRR 185 (B4) and 277146847 - CRC 1238

Table I. Table of ranks for parallel and antiparallel boundary spins computed from the stochastic matrix $F_{\alpha \beta}$ for different system sizes $N$. Note that only $\varphi<\pi$ are considered because of the NESS symmetry $\varphi \rightarrow-\varphi$. We also show the degeneracy of the eigenvalue $\lambda_{0}=(N-1) J \Delta$ of $h_{D}$. The last three columns check conditions A, B and C of the criterion for rank 2 states. Symbols $\checkmark, \boldsymbol{X}$, N/A and "-" indicate, respectively, that the corresponding property is satisfied, violated, nonapplicable, and noncheckable. Property $\mathrm{C}$ should only be checked in case of extra degeneracy of $\lambda_{0}>2$. For $N \geq 13$ the NESS rank (whenever it is larger than 2) cannot be determined reliably because of numerical precision-related issues [31]

\begin{tabular}{cccccccc}
\hline \hline$N$ & $\varphi$ & NESS rank Full rank $\operatorname{deg}\left(\lambda_{0}\right)$ & A & B & C \\
\hline 3 & $\frac{\pi}{2}$ & 2 & 2 & 2 & $\checkmark$ & N/A & N/A \\
4 & $\frac{\pi}{3}, \frac{2 \pi}{3}$ & 2 & 4 & 2 & $\checkmark$ & $\checkmark$ & N/A \\
5 & $\frac{\pi}{2}$ & 8 & 8 & 4 & $\checkmark$ & $x$ & $\checkmark$ \\
& $\frac{\pi}{4}, \frac{3 \pi}{4}$ & 2 & & 2 & $\checkmark$ & $\checkmark$ & N/A \\
6 & $\frac{\pi}{5}, \ldots, \frac{4 \pi}{5}$ & 2 & 16 & 2 & $\checkmark$ & $\checkmark$ & N/A \\
7 & $\frac{\pi}{2}$ & 32 & 32 & 8 & $\checkmark$ & $x$ & $\checkmark$ \\
& $\frac{\pi}{3}, \frac{2 \pi}{3}$ & 22 & & 4 & $\checkmark$ & $\checkmark$ & $x$ \\
& $\frac{\pi}{6}, \ldots, \frac{5 \pi}{6}$ & 2 & & 2 & $\checkmark$ & $\checkmark$ & N/A \\
8 & $\frac{\pi}{7}, \ldots, \frac{6 \pi}{7}$ & 2 & 64 & 2 & $\checkmark$ & $\checkmark$ & N/A \\
9 & $\frac{\pi}{2}$ & 128 & 128 & 16 & $\checkmark$ & $x$ & $\checkmark$ \\
& $\frac{\pi}{4}, \frac{3 \pi}{4}$ & 72 & & 4 & $\checkmark$ & $x$ & $\checkmark$ \\
& $\frac{\pi}{8}, \frac{3 \pi}{8}, \frac{5 \pi}{8}, \frac{7 \pi}{8}$ & 2 & & 2 & $\checkmark$ & $\checkmark$ & N/A \\
10 & $\frac{\pi}{3}, \frac{2 \pi}{3}$ & 170 & 256 & 8 & $\checkmark$ & $\checkmark$ & $x$ \\
& $\frac{\pi}{9}, \frac{2 \pi}{9}, \frac{4 \pi}{9}, \frac{5 \pi}{9}, \frac{7 \pi}{9}, \frac{8 \pi}{9}$ & 2 & & 2 & $\checkmark$ & $\checkmark$ & N/A \\
11 & $\frac{\pi}{2}$ & 512 & 512 & 32 & $\checkmark$ & $x$ & $\checkmark$ \\
& $\frac{\pi}{5}, \frac{2 \pi}{5}$ & 254 & & 4 & $\checkmark$ & $\checkmark$ & $x$ \\
& $\frac{\pi}{10}, \frac{3 \pi}{10}, \frac{7 \pi}{10}, \frac{9 \pi}{10}$ & 2 & & 2 & $\checkmark$ & $\checkmark$ & N/A \\
12 & $\frac{\pi}{11}, \ldots, \frac{10 \pi}{11}$ & 2 & 1024 & 2 & $\checkmark$ & $\checkmark$ & N/A \\
13 & $\frac{\pi}{2}$ & 2048 & 2048 & - & - & - & - \\
& $\frac{\pi}{3}, \frac{2 \pi}{3}$ & - & & 16 & $\checkmark$ & $\checkmark$ & $x$ \\
& $\frac{\pi}{4}, \frac{3 \pi}{4}$ & - & & 8 & $\checkmark$ & $x$ & $\checkmark$ \\
& $\frac{\pi}{6}, \frac{5 \pi}{6}$ & - & & 4 & $\checkmark$ & $x$ & $\checkmark$ \\
& $\frac{\pi}{12}, \frac{5 \pi}{12}, \frac{7 \pi}{12}, \frac{11 \pi}{12}$ & 2 & & 2 & $\checkmark$ & $\checkmark$ & N/A \\
\hline \hline
\end{tabular}

(C05) and under Germany's Excellence Strategy Cluster of Excellence Matter and Light for Quantum Computing (ML4Q) EXC 2004/1 390534769 and from the European Research Council (ERC) under the Horizon 2020 research and innovation programme, grant agreement No. 648166 (Phonton) and No. 694544 (OMNES), and from interdisciplinary UoC Forum "Classical and quantum dynamics of interacting particle systems" is gratefully acknowledged. We thank G. Schütz for critical remarks on the paper. 
[1] A. D. E. De Moivre, The Doctrine of Chances; or, A Method for Calculating the Probabilities of Events in Play (Pearson, London, 1718).

[2] B. Misra and E. C. G. Sudarshan, "The Zeno's paradox in quantum theory", Journal of Mathematical Physics 18, 756 (1977).

[3] C. Presilla, R. Onofrio, U. Tambini, "Measurement quantum mechanics and experiments on quantum Zeno effect", Ann. Phys. (N.Y.) 248, 95-121 (1996).

[4] K. Koshino, and A. Shimizu, "Quantum Zeno effect by general measurements", Physics Reports 412, 191 (2005).

[5] H.-P. Breuer and F. Petruccione, The Theory of Open Quantum Systems (Oxford University Press, Oxford, 2002).

[6] P. Zanardi and L. Campos Venuti, "Coherent Quantum Dynamics in Steady-State Manifolds of Strongly Dissipative Systems", Phys. Rev. Lett. 113, 240406 (2014).

[7] J. Marshall, L. Campos Venuti, and P. Zanardi, "Modular quantum-information processing by dissipation", Phys. Rev. A 94, 052339 (2016).

[8] W. M. Itano, D.J . Heinzen, J. J. Bollinger, and D. J. Wineland, "Quantum Zeno effect", Phys. Rev. A 41, 2295 (1990).

[9] P. Kwiat, H. Weinfurter, T. Herzog, A. Zeilinger, and M. A. Kasevich, "Interaction-free measurement", Phys. Rev. Lett. 74, 4763 (1995).

[10] A. Signoles, A. Facon, D. Grosso, I. Dotsenko, S. Haroche, J.M. Raimond, M. Brune, and S. Gleyzes, "Confined quantum Zeno dynamics of a watched atomic arrow", Nature Physics 10, 715 (2014).

[11] F. Schäfer, I. Herrera, S. Cherukattil, C. Lovecchio, F. S. Cataliotti, F. Caruso, and A. Smerzi, "Experimental realization of quantum zeno dynamics", Nature Communications 5, 3194 (2014).

[12] Y. S. Patil, S. Chakram, and M. Vengalattore, "Measurementinduced localization of an ultracold lattice gas", Phys. Rev. Lett. 115, 140402 (2015).

[13] E. W. Streed, J. Mun, M. Boyd, G. K. Campbell, P. Medley, W. Ketterle, and D. E. Pritchard, "Continuous and Pulsed Quantum Zeno Effect”, Phys. Rev. Lett. 97260402 (2006).

[14] A. Beige, D. Braun, B. Tregenna, and P. L. Knight, "Quantum computing using dissipation to remain in a decoherence-free subspace", Phys. Rev. Lett. 851762 (2000).

[15] C.-E. Bardyn, M. A. Baranov, C. V. Kraus, E. Rico, A. Imamgolu, P. Zoller, and S. Diehl, "Topology by dissipation”, New Journal of Physics 15, 085001 (2013).

[16] K. Stannigel,P. Hauke, D. Marcos, M. Hafezi, S. Diehl, M. Dalmonte, and P. Zoller, "Constrained Dynamics via the Zeno Effect in Quantum Simulation: Implementing Non-Abelian Lattice Gauge Theories with Cold Atoms", Phys. Rev. Lett. 112, 120406 (2014).

[17] F. Verstraete, M. M. Wolf, and J. Ignacio Cirac, "Quantum computation and quantum-state engineering driven by dissipation",
Nature Physics 5, 633 (2009).

[18] W. Yi, S. Diehl, A.J. Daley, and P. Zoller, "Driven-dissipative many-body pairing states for cold fermionic atoms in an optical lattice", New Journal of Physics 14, 055002 (2012).

[19] T. J. Elliott, W. Kozlowski, S. F. Caballero-Benitez, and I. B. Mekhov, "Multipartite Entangled Spatial Modes of Ultracold Atoms Generated and Controlled by Quantum Measurement”, Phys. Rev. Lett. 114, 113604 (2015).

[20] K. Winkler, G. Thalhammer, F. Lang, R. Grimm, J. Hecker Denschlag, A. J. Daley, A. Kantian, H. P. Büchler, and P. Zoller, "Repulsively bound atom pairs in an optical lattice", Nature (London) 441, 853 (2006).

[21] P. Zanardi, J. Marshall, and L. Campos Venuti, "Dissipative universal Lindbladian simulation”, Phys. Rev. A 93, 022312 (2016).

[22] V. Popkov, S. Essink, C. Presilla, and G. M. Schütz, "Effective quantum Zeno dynamics in dissipative quantum systems ", Phys. Rev. A 98, 052110 (2018).

[23] J. .G. Kemeny, J. L. Snell, and A. W. Knapp, "Denumerable Markov Chains: with a chapter of Markov Random Fields" by David Griffeath (Graduate Texts in Mathematics) (Springer) 2012.

[24] G. Schaller, Open Quantum Systems Far From Equilibrium (Springer, Heidelberg, 2014).

[25] P. Zanardi and L. Campos Venuti, "Geometry, robustness, and emerging unitarity in dissipation-projected dynamics", Phys. Rev. A 91, 052324 (2015).

[26] V. Popkov, C. Presilla, and J. Schmidt, "Targeting pure quantum states by strong noncommutative dissipation”, Phys. Rev. A 95, 052131 (2017).

[27] G. T. Landi, E. Novais, M. J. de Oliveira, and D. Karevski, "Flux rectification in the quantum $X X Z$ chain", Phys. Rev. E 90, 042142 (2014).

[28] V. Popkov, J. Schmidt, and C. Presilla, "Spin-helix states in the XXZ spin chain with strong boundary dissipation ", Journal of Physics A: Mathematical and Theoretical, 50, 435302 (2017).

[29] V. Popkov and C. Presilla, "Obtaining pure steady states in nonequilibrium quantum systems with strong dissipative couplings ”, Phys. Rev. A 93, 022111 (2016).

[30] S. Essink, S. Wolff, G. M. Schütz, C. Kollath, and V. Popkov, "Transition between dissipatively stabilized helical states", Phys. Rev. Research 2, 022007(R), 2020.

[31] S. Essink, master's thesis, Universität Bonn, April, 2018.

[32] M. Raghunandan, F. Wolf, C. Ospelkaus, P. O. Schmidt, H. Weimer, "Initialization of quantum simulators by sympathetic cooling", Science Advances, 6, eaaw9268, 2020.

[33] F. Tacchino, A. Chiesa, S. Carretta, D. Gerace, "Quantum computers as universal quantum simulators: state-of-the-art and perspectives", Advanced Quantum Technologies 3, 1900052, 2020. 